\title{
VALIDATION OF A BRAKE MONITORING SYSTEM USING A MULTI-AXLE ROLLER-RIG
}

\author{
NICOLA BOSSO*, MATTEO MAGELLI ${ }^{\dagger} \&$ NICOLÒ ZAMPIERI ${ }^{\ddagger}$ \\ Department of Mechanical and Aerospace Engineering, Politecnico di Torino, Italy
}

\begin{abstract}
A widespread diffusion of monitoring systems is the key to improving safety and reliability of railway vehicles and to reducing maintenance costs by increasing the lifetime of structural and mechanical components. Several on-board devices have been conceived in recent years to detect faults on critical parts, by monitoring the real-time conditions of wheelsets, bearings, brakes, bogie, carbody, etc. These systems rely on powered sensor nodes, mounted on different parts of the vehicle, which acquire signals of interest and use proper algorithms to detect component faults or train derailments. Roller-rigs represent a good solution to develop and validate new monitoring devices with good repeatability of test conditions. The research group from Politecnico di Torino realized an innovative 1:5 scaled roller-rig, consisting of four wheelsets running on the same pair of rollers, originally used for investigations on wheel-rail adhesion. Then, minor changes were made to simulate and test a braking system monitoring device. Each wheelset is provided with a braking system, consisting of two brake discs and two brake callipers, while the rollers are powered using one AC brushless motor. A freight train pneumatic system is reproduced thanks to three electropneumatic regulators, which simulate the pressure in brake pipe, auxiliary reservoir and brake cylinder. The test bench and the motor drive are managed by an industrial PC using the software LabVIEW, allowing to perform braking operations of a real vehicle. Sensors are installed to measure wheelset speed, calliper temperature and pressures in the braking system. These signals are sent to an electronic control unit, which could be provided with a fault detection logic. The paper deals with the experimental setup of the bench and the preliminary results obtained by laboratory tests, which highlighted that the apparatus can be a reliable tool to develop new monitoring algorithms for train braking systems.
\end{abstract}

Keywords: roller-rig, railway monitoring, braking system, fault detection, railway brake plant simulation.

\section{INTRODUCTION}

Safety and reliability of railway vehicles can be improved by the adoption of monitoring systems able to detect anomalies in vehicle running behaviour and faults of the critical components of the bogie. Usually the maintenance of the vehicle components is scheduled considering a fixed inspection period. This approach is simple and easy to schedule, but it does not allow to optimize costs and safety. In fact, failures and malfunctions could occur in advance of the subsequent inspection, while other inspections could take place when not necessary. The adoption of specific monitoring systems to check the condition of critical components represent a valid solution able to improve the predictive maintenance interventions based on data recorded during the vehicle operation.

Monitoring systems currently available can be divided into two main categories according to the installation site: on-track systems and on-board systems (installed on the vehicle). The first solution has several advantages such as easy installation and available power supply. However, this solution does not guarantee a continuous monitoring of the railway vehicle, since detections are performed only when the vehicle runs on track sections where the

\footnotetext{
* ORCID: http://orcid.org/0000-0002-5433-6365

† ORCID: http://orcid.org/0000-0002-2962-7873

* ORCID: http://orcid.org/0000-0002-9197-1966
} 
monitoring system is installed. On the other hand, on-board systems assure a continuous monitoring, and are therefore capable to detect any faults and failures as soon as they occur. The main drawback of on-board systems is the more complex implementation and assembly, since the available space on the vehicle is extremely limited. Furthermore, the main obstacle to the spread of on-board systems is the difficulty in supplying electrical power to the sensors and control units. In fact, normally the freight wagons do not have an electrical power supply, but even where the electrical power is available, the wiring for all the devices distributed along the train is difficult to realize in compliance with current standards [1]. However, recent advances in the field of energy harvesting techniques [2], [3], which allow to obtain electricity from environmental energy, could be an interesting opportunity for the development of on-board monitoring systems. The state of the art proposed by Bernal et al. [4] shows that monitoring systems are composed by sensors for measuring quantities of interest (accelerometers, thermocouples, thermistors, etc.), devices for signal conditioning and acquisition, and control units running specific algorithms [5], [6] able to detect anomalies. To increase the safety level of railway vehicles, great interest is obviously directed to the development of monitoring systems for the brake system, such as the one proposed by Somà et al. [7], Zampieri et al. [8] and by Bosso et al. [9].

The validation of new monitoring systems and related algorithms for data analysis requires extensive experimental test campaigns, typically carried out on track, with high costs, line occupation and poor repeatability of operating conditions. A valid alternative to on-track tests is the use of roller-rigs [10], i.e., test devices, in which the track is replaced by pairs of rollers. This solution allows to reproduce with good accuracy the geometry and the kinematics of the wheel-rail contact, as well as the dynamics of the railway vehicle [11]. The aim of the paper is to demonstrate how the innovative 1:5 scale multi-axles roller-rig, designed by the research team of the Politecnico di Torino, can be adopted to test and validate monitoring systems of the braking system. The experimental system, proposed in this work, can in fact be considered a Hardware In the Loop (HIL) system of the braking system of a railway vehicle.

The braking manoeuvre is replicated on the roller-rig by means of a braking system, and it is managed by a control algorithm developed in LabVIEW. Sensors are installed on the bench in order to measure the quantities of interest and their signals are acquired by the monitoring unit. The acquired signals are processed by specific algorithms in order to evaluate the condition of the braking system. The architecture proposed in this work will allow us to develop and validate braking system monitoring algorithms without requiring on-track tests, at least during the early stages of development. The paper shows, both the mechanical design of the test bench and the pneumatic system designed to replicate the braking system of a freight vehicle.

\section{DESCRIPTION OF THE MULTI-AXLE ROLLER-RIG}

The multi-axle roller-rig, shown in Fig. 1, is an evolution of the single axis roller-rig, already designed by the authors which was previously designed and used for the study of degraded adhesion phenomena [12], [13] and wear [14]. More recently the attention of the research group focused on the adhesion recovery phenomenon [15]. At first, the research team tried to study this phenomenon adopting a traditional single axis roller-rig [16], which is composed by a single wheelset suspended on a pair of rollers. The results show that the inefficiencies of the system used to remove the contaminant made the experimental results difficult to interpret. Therefore, the innovative 1:5 scaled multi-axle roller-rig was designed and realized [17], [18]. The peculiarity of the testing device is to dispose of four wheelsets running on the same pair of rollers. This solution is in fact essential to correctly study and reproduce the 


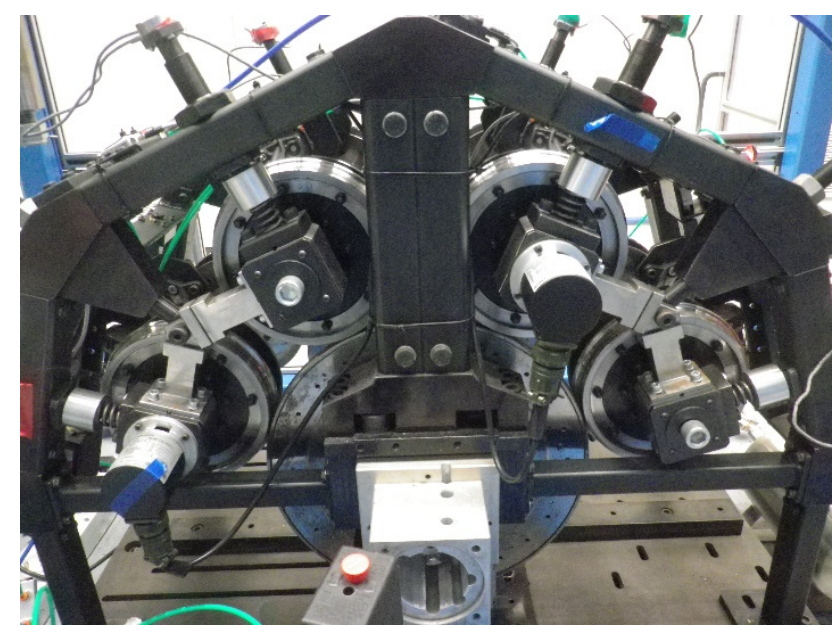

Figure 1: Lateral view of the multi-axle roller-rig.

different levels of adhesion of the wheelsets of a vehicle when running on a contaminated section of rail. The test bench has been designed according to the Jaschinski's similitude, which keeps a 1:1 scale for the accelerations.

\subsection{Mechanical design of the roller-rig}

Considering the mechanical arrangement, the roller-rig is composed by the frame, two rollers and four 1:5 scaled wheelsets. The two rollers, in this case secured each other thanks to a rigid joint, are driven by a single six poles brushless motor, controlled by means of a digital drive. The rollers consist of a disk to which a rim is fixed. The rim is machined to realize a 1:5 scaled UIC60 rail profile canted 1:20. Each wheelset is composed by an axle on which are fixed two brake discs and two 1:5 scaled wheels. The last ones consist of a disk and an external rim, which is machined to obtain the $\mathrm{S} 1002$ wheel profile. The nominal diameter of the wheels $d_{W}$ and the rollers $d_{R}$ are respectively $184 \mathrm{~mm}$ and $368 \mathrm{~mm}$.

The two axle-boxes, installed at the extremities of each wheelset, are rigidly connected to the frame in the longitudinal and lateral direction while the flexibility in the vertical direction is guaranteed by two helical springs (one on each side) which act as primary suspension. The preload generated by each spring can be independently regulated thanks to a dedicated screw loading system. In this way, it is possible to simulate different axle-load values and compensate for the differences in the normal component of the weight force due to the inclination of the wheelsets with respect to the vertical direction.

In order to perform the braking tests and testing the monitoring system, the test bench is equipped with several sensors able to measure the quantities of greatest interest and an industrial PC (NI PXIe-8840) to control the braking manoeuvre. All the axle-boxes are equipped with load button cells, with a maximum load of $1000 \mathrm{lb}(454 \mathrm{~kg})$ and a resolution of $0.5 \mathrm{lb}(0.227 \mathrm{~kg})$, for regulating the wheel load. During the braking tests, however, only the signal generated by the load cell installed on one wheelset is used. In fact, the freight wagons are usually equipped with weighting cells, able to estimate the weight of the entire bogie from the load generated by one of the 8 springs composing the primary suspension of the bogie. However, since each axle-box of test bench is equipped with a single spring, the 
axle-load $\mathrm{Q}$ is estimated starting from the signal $\mathrm{F}_{\mathrm{S}}$ generated by one of the two load cells. The eqn (1) is used to calculate the axle load and $\mathrm{m}$ is the wheelset mass $(15.16 \mathrm{~kg})$ and $\mathrm{g}$ is the gravity acceleration

$$
Q=2 F_{S}+m g \cos 72^{\circ} \text {. }
$$

During the experimental tests shown in this paper only one wheelset is braked. The braking torque is measured by two S-shaped load cells, with a maximum load of $300 \mathrm{lb}$ $(136 \mathrm{~kg})$ and resolution of $0.15 \mathrm{lb}(0.07 \mathrm{~kg})$. The load cells measure the reaction forces of the brackets supporting the two brake callipers. The breaking torque $\mathrm{C}_{B}$ acting on the wheelset can be estimated according to eqn (2).

$$
C_{B}=\left(F_{B, 1}+F_{B, 2}\right) \cdot r_{B}
$$

In eqn (2), $F_{B, 1}$ and $F_{B, 2}$ are the braking force values measured by the load cells, while $\mathrm{r}_{B}$ is the equivalent braking radius, which has been estimated during the calibration procedure of the test bench and is equal to $79.24 \mathrm{~mm}$. The wheelset is also equipped with an incremental encoder (resolution of $1024 \mathrm{ppr}$ ), which allows to obtain the angular position of the wheelset, from which the speed is calculated by numerical differentiation.

Three proportional pneumatic valves, which regulate the outlet pressure in the range 0-9 bar against a voltage signal $0-10 \mathrm{~V}$, are used to simulate the braking system of the vehicle. One valve simulates the pressure in the brake pipe, another one regulates the pressure to the auxiliary reservoir and the last one allows to supply the desired pressure value to the brake cylinder. The three valves receive as input a reference voltage signal (set), and suitably regulate the outlet pressure, supplying the feedback pressure value as a voltage signal in the range $1-5 \mathrm{~V}$.

The experimental setup includes two Pt1000 thermistors used to measure the calliper body temperature. The sensors perform temperature measurements in the range $-50^{\circ} \mathrm{C}$ to $300^{\circ} \mathrm{C}$ and have a maximum resistance error of $1.2 \Omega$.

\subsection{Design of the pneumatic braking system}

In order to test new prototypes of monitoring systems for freight vehicle brakes, the multiaxles roller-rig is equipped with a braking system that replicates the one normally installed on freight trains [19], [20]. Fig. 2 shows the architecture of the typical braking system of a freight railway vehicle.

Under normal operating conditions the brake pipe is maintained at a pressure of 5 bar. The train driver can change the pressure of the brake pipe by acting on the brake valve (V), which receives compressed air from the main reservoir (MR), kept by the locomotive compressor at a pressure of about 8 bar. Each wagon is equipped with a triple valve (D), which is the decision-making element of the braking manoeuvre. This valve compares the pressure in the pipeline with the pressure in the control tank (CR). When the driver activates the braking operation the pressure in the brake pipe drops below 5 bar, and the triple valve connects the auxiliary reservoir (AR), at the pressure of 3.8 bar, and the brake cylinder chamber. Traditional freight trains are equipped with a single brake cylinder for bogie. The force generated by the cylinder is increased by means of a linkage which allows to press each pair of brake pads against the respective wheel. Emergency braking differs from service one since it generates a fast discharge of the brake pipe and it is not reversible, i.e., once the manoeuvre is activated, the pipeline is completely discharged. In contrast, at the end of a service braking, the release of the brake cylinder requires that the triple valve connects the brake cylinder 


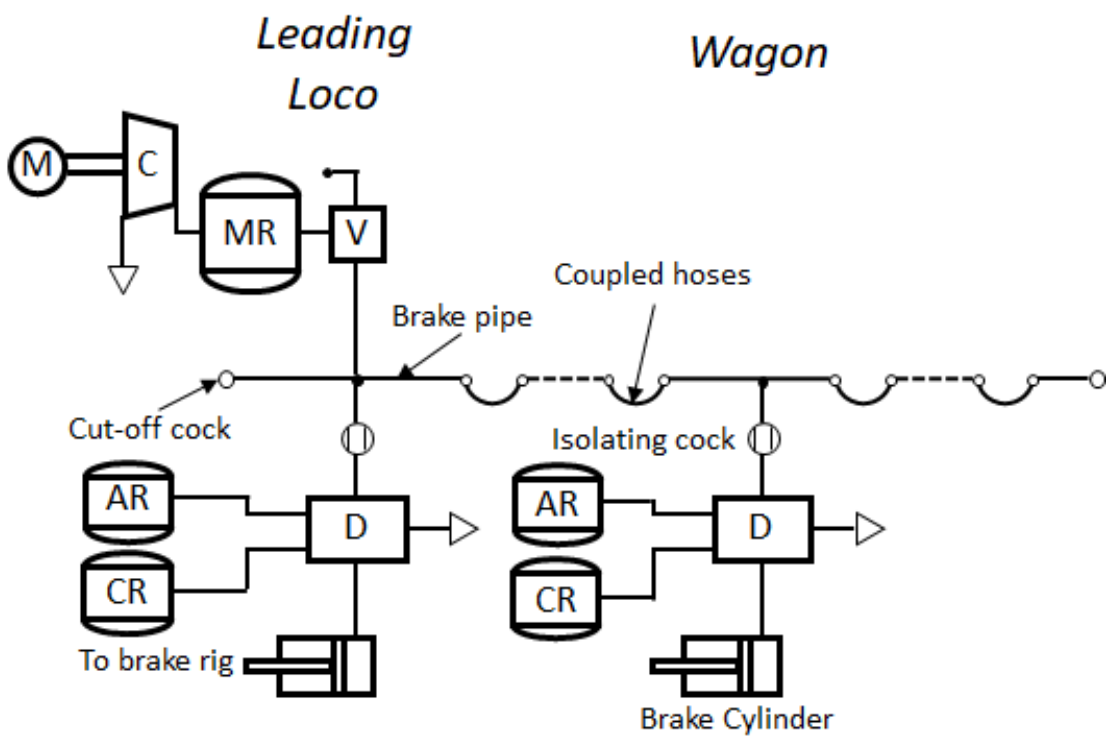

Figure 2: Typical architecture of the braking system of a railway freight vehicle.

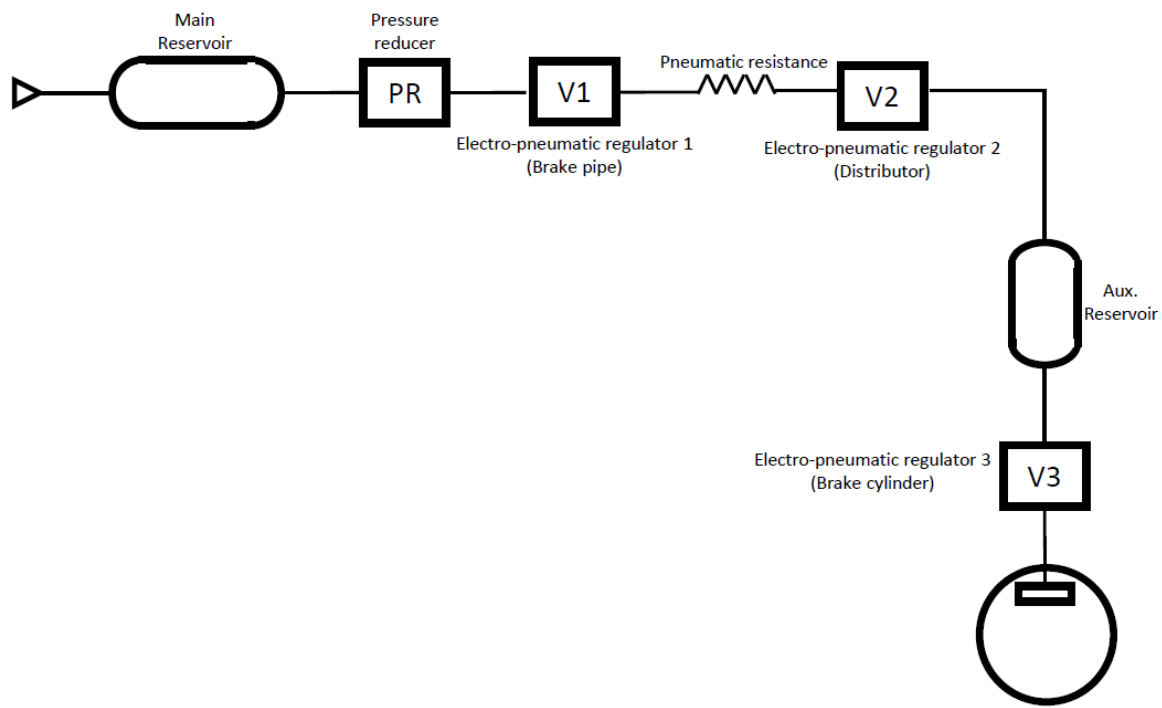

Figure 3: Architecture of the braking system adopted for the multi-axle roller-rig.

chamber and the atmosphere, while the auxiliary reservoir is refilled by the brake pipe at the pressure of 3.8 bar.

The architecture of the braking system built on the test bench to replicate the real one is shown in Fig. 3. 
The compressed air, supplied by an industrial compressor, is stocked in the main reservoir (MR). Downstream the compressor there is a pressure regulator which maintains a pressure of 8 bar upstream of the first proportional valve (EP Reg. 1). The output of this valve reproduces the brake pipe, and in the absence of braking effort the pressure is kept at 5 bar. Downstream of the first proportional valve there is a long pipe, which simulates a distributed resistance and allows to physically reproduce the propagation delay of the pressure wave along the brake pipe. The output of the second proportional valve (E-P Reg. 2), set at 3.8 bar, is connected to a tank that simulates the auxiliary reservoir. This one supplies the third proportional valve (E-P Reg. 3), which regulates the pressure of the two brake callipers installed on the wheelset, replacing the distributor. The pressure downstream of the third proportional valve, which represents the pressure at the brake cylinder, is regulated as a function of the pressure drop detected in the brake pipe, according to the transfer function shown in Fig. 4.

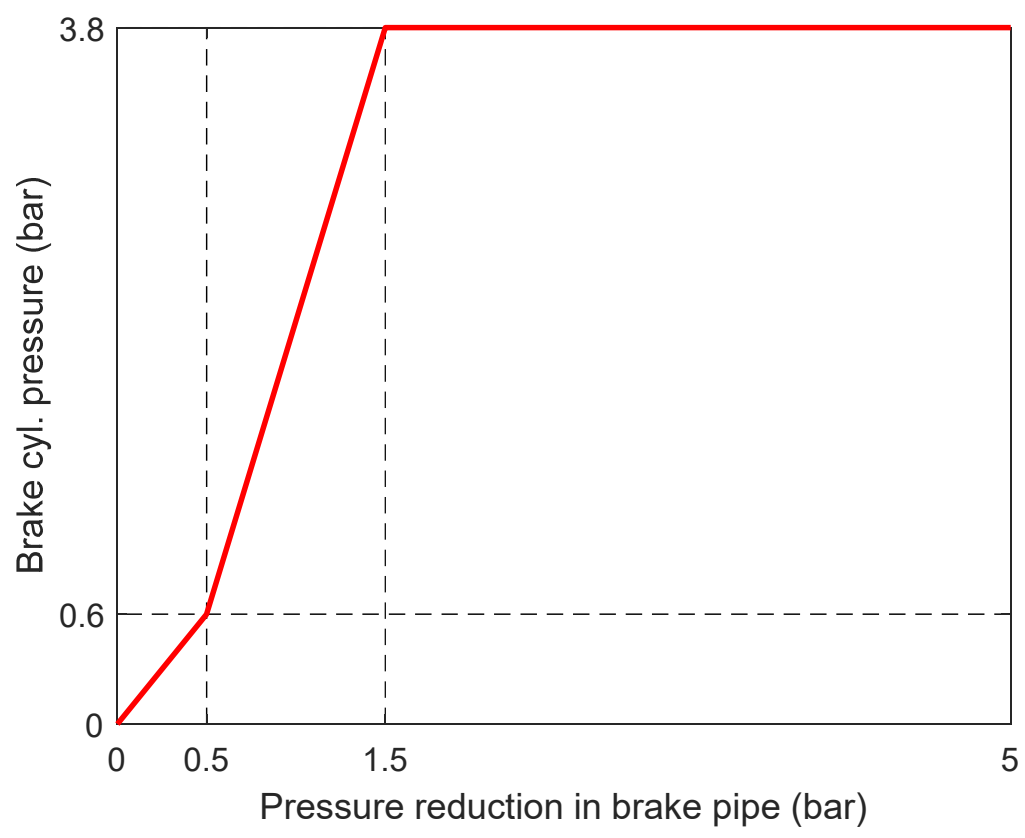

Figure 4: Transfer function of the brake cylinder pressure as a function of the pressure drop in the brake pipe.

The transfer function has been defined according to the following considerations. First, the indications of UIC 540 [20] standard have been followed, which suggests that the maximum pressure to the brake cylinder ( 3.8 bar) should occur in correspondence of a pressure drop of 1.5 bar of the brake pipe. Furthermore, experimental tests on the roller-rig show that the pads exert a braking effort on the disc for pressure value higher than 0.6 bar. In order to considering this effect a brake pressure reduction of 0.5 bar has been considered for the definition of the transfer function. In this way, when the braking manoeuvre starts, pressurized air flows to the brake cylinder, keeping the pads close to the disc as soon as the brake pipe pressure drops. 


\section{SIMULATION OF THE BRAKING MANEUVER}

The simulation of realistic braking manoeuvres on the test bench is managed by a dedicated control software (LabVIEW VI), developed in LabVIEW 2016 and implemented in the industrial PC. The analogue output (AO) module NI-9263 (4 channels, 16 bits resolution and maximum sampling rate of $100 \mathrm{kS} / \mathrm{s} / \mathrm{ch}$ ) is used to adjust the outlet pressure of the 3 electro-pneumatic regulators, which reproduce the brake pipe, the distributor and the brake cylinder. When no braking effort is applied, the 3 set values are equal to 5, 3.8 and 0 bar respectively. The feedback values are measured by the electro-pneumatic regulators and acquired through an USB analogue input (AI) module NI-9239 (4 channels, 24 bits resolution and maximum sampling rate equal to $50 \mathrm{kS} / \mathrm{s} / \mathrm{ch}$ ). The tangential forces measured by the two S-beam load cells installed on the first wheelset and the normal force measured by the button load cell mounted on the axle box on the non-powered side of the same wheelset are acquired by the strain bridge input module NI PXIe-4330, with 8 channels, resolution equal to 24 bits and maximum sampling rate equal to $25.6 \mathrm{kS} / \mathrm{s} / \mathrm{ch}$. A servo mode control is performed on the brushless motor powering the rollers, with the reference speed being an analogue signal coming from the AO module PXIe-4322, which also generates three analogue signals corresponding to the feedback pressure at the outlet of the electro-pneumatic regulators and an analogue signal reproducing the weighing cell output.

These analogue output signals, as well as the signals from the encoder mounted on wheelset 1 and the measurements from the Pt1000 thermistors installed on the brake pads are acquired by the monitoring unit, which was used by the research team in the past to develop and test a monitoring system able to control the vehicle running behaviour and to diagnostic the condition of the main parts of the bogie [21]. The monitoring unit is managed by a 32-bit ARM7 processor, provided with a multichannel synchronous acquisition system with proper signal conditioning depending on the type of acquired signal (ICP accelerometers, encoders, thermocouples and thermistors). Data acquisition and transfer operations, which can be performed by means of the TCP/IP protocol or a CAN network, are supervised by an editable built-in resident code. The vehicle condition parameters and possible anomalies can be stored in a SD card included in the monitoring unit. Data operations are performed by an embedded PC which is connected to the monitoring unit TCP/IP network. A single embedded PC could be used to managed data from several monitoring units, however the typical arrangement comprises a monitoring unit mounted on each bogie and an embedded PC for each vehicle.

In order to replicate a braking manoeuvre on the multi-axle roller-rig, it is essential to supply compressed air to the third electro-pneumatic regulator, representing the brake cylinder, and to simulate the vehicle inertia on the roller shaft. Focusing on the longitudinal dynamics of a railway vehicle [22]-[24] and assuming that coupling systems are infinitely stiff so that the entire train moves as a single rigid body and slows down with a deceleration $\ddot{\mathrm{x}}$, the equilibrium in the longitudinal direction is expressed through eqn (3)

$$
\sum_{j=1}^{N_{V}} \sum_{i=1}^{N_{W, j}} F_{X, j i}=\sum_{j=1}^{N_{V}} \sum_{i=1}^{N_{W, j}} M_{, j i} \ddot{x}-\sum_{j=1}^{N_{V}} F_{r e s, j} .
$$

In eqn (3), $F_{x}$ is the longitudinal force acting on each wheelset-rail interface, $M$ is the axle load (in $\mathrm{kg}$ ), $\mathrm{F}_{\text {res }}$ is the total retarding force on each vehicle, subscript $\mathrm{j}$ refers to each of the $\mathrm{N}_{\mathrm{V}}$ vehicles composing the train, while subscript $\mathrm{i}$ is related to the number of wheelset $\mathrm{N}_{\mathrm{W}}$ of each vehicle. Fig. 5(a) shows the forces acting in the longitudinal direction on the $\mathrm{j}^{\text {th }}$ vehicle in the train composition. 


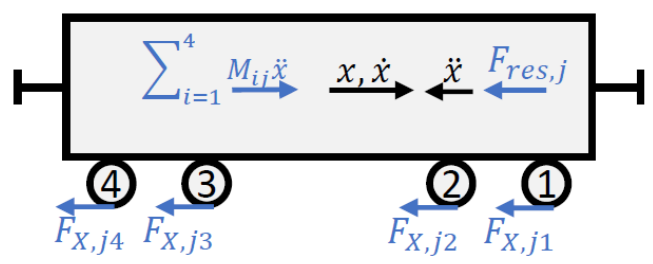

(a)

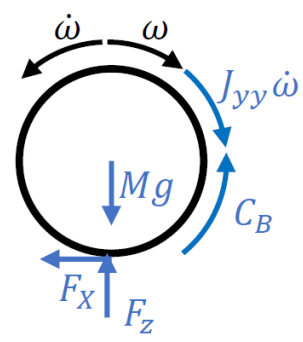

(b)

Figure 5: Force equilibriums. (a) Equilibrium in the longitudinal direction; (b) Rotational equilibrium of the wheelset.

On the hypothesis that the vehicle is running on a straight track with no grade and curves, that the force acting at each wheel-rail interface is the same for all wheelsets and neglecting the aerodynamic resistances, the longitudinal equilibrium can be simplified, as eqn (4) states

$$
F_{X}=M \ddot{x}
$$

The total longitudinal force acting on each wheelset can be calculated from the rotational equilibrium of the wheelset, expressed by eqn (5) and shown in Fig. 5(b), where $\mathrm{C}_{B}$ is the total braking force, $\mathrm{r}_{W}$ is the wheel radius, $\dot{\omega}$ is the wheel deceleration and $\mathrm{J}_{y y}$ is the total inertia of the wheelset

$$
F_{X}=\frac{C_{B}-J_{y y} \dot{\omega}}{r_{W}} .
$$

If perfect adhesion conditions are assumed, the relationship between the angular deceleration of the wheelset and the vehicle longitudinal deceleration is expressed by eqn (6), and the longitudinal deceleration can be calculated from eqn (7)

$$
\begin{gathered}
\dot{\omega}=\frac{\ddot{x}}{r_{W}}, \\
\ddot{x}=\frac{C_{B} r_{W}}{M r_{W}^{2}+J_{y y}} .
\end{gathered}
$$

Since the bench is realized following Jaschinski's similitude model, which guarantees that the acceleration of the scaled system is equal to the acceleration of the full-scale system, equation (NN) is valid also using the scaled quantities. The braking torque is obtained from the two load cells installed on wheelset 1 , while the wheelset mass is calculated from the button load cell installed on the axle-box of the non-powered side of wheelset 1 . The total inertia of the wheelset is estimated from the CAD model of the wheelset and equal to $0.051 \mathrm{~kg} \cdot \mathrm{m}^{2}$. 
Therefore, at each time step of the simulation of the braking manoeuvre the LabVIEW VI can calculate a value for the vehicle deceleration, and an analogue signal is sent to the motor drive in order to set the proper motor speed.

\section{EXPERIMENTAL SETUP AND RESULTS}

At this first stage of the work, preliminary tests were performed to assess the capability of the new bench design to simulate realistic braking manoeuvres. Simple experimental tests were carried out by imposing a ramp of pressure reduction in the electro-pneumatic regulator reproducing the brake pipe, and correspondingly increasing the brake cylinder pressure according to the feedback value of the brake pipe pressure. The brake pipe was not totally released, but the pressure ramp decrease ramp was stopped at a limit value. Three tests were performed in order to investigate the influence of both the pressure ramp and the limit value. Table 1 summarizes the values of the main parameters of the 3 experimental braking tests, i.e., the maximum pressure reduction in the brake pipe, the corresponding maximum pressure in the brake cylinder and a ramp slope parameter. The latter is the time that the ramp signal would require to reach a pressure decrease equal to 1.5 bar in the brake pipe, if no previous saturation was superimposed. As shown in Table 1, the same ramp signal was used for tests 1 and 2, but a lower saturation level for the brake pipe pressure was superimposed in test 2, thus leading to a lower maximum pressure in the brake cylinder. The ramp used in tests 1 and 2 is such that a maximum pressure reduction of 1.5 bar in the brake pipe, corresponding to a maximum brake cylinder pressure equal to 3.8 bar, would take $18 \mathrm{~s}$, which is the same as the time usually needed in $G$ regime to reach the maximum braking pressure of 3.8 bar in the brake cylinder. On the other hand, test 3 was performed by superimposing the same limit value for the brake pipe reduction as test 1 , thus leading to the same maximum brake cylinder pressure, but a faster ramp signal was generated. In all tests, the brake forces measured by the S-beam cells were acquired by the LabVIEW VI and used to estimate the deceleration of the vehicle, thus modifying accordingly the set value for the roller speed and reproducing the vehicle inertia.

Table 1: Main parameters for experimental braking tests.

\begin{tabular}{|c|c|c|c|c|}
\hline \multirow[t]{2}{*}{ Test } & \multirow{2}{*}{$\begin{array}{l}\text { Limit value of pressure } \\
\text { reduction in brake pipe } \\
\text { (bar) }\end{array}$} & \multirow{2}{*}{$\begin{array}{c}\text { Max. brake } \\
\text { cylinder pressure } \\
\text { (bar) }\end{array}$} & \multicolumn{2}{|c|}{$\begin{array}{l}\text { Time for } 1.5 \text { bar pressure } \\
\text { reduction in brake pipe (s) }\end{array}$} \\
\hline & & & Scaled & Real vehicle \\
\hline 1 & 0.71 & 1.16 & 8 & 18 \\
\hline 2 & 0.66 & 1 & 8 & 18 \\
\hline 3 & 0.71 & 1.16 & 6.5 & 14.5 \\
\hline
\end{tabular}

Fig. 6 shows the roller peripheral speed as a function of time (up) and the braking curves (down), i.e., the speed vs position curve, obtained in the three tests. Please note that the plots refer to scaled values of time, position and speed. The initial roller speed in all three tests is set to about $260 \mathrm{rpm}$, corresponding to a peripheral speed of $5 \mathrm{~m} / \mathrm{s}$ and an initial speed for a real vehicle of $40 \mathrm{~km} / \mathrm{h}$.

The braking manoeuvres starts as soon as a pressure reduction is imposed in the brake pipe, but in all three tests a few seconds elapse before the rollers start to slow down, since no braking effort is applied when the brake cylinder pressure is below a certain threshold, experimentally determined to be equal to about 0.6 bar. It can be noticed that this time interval 

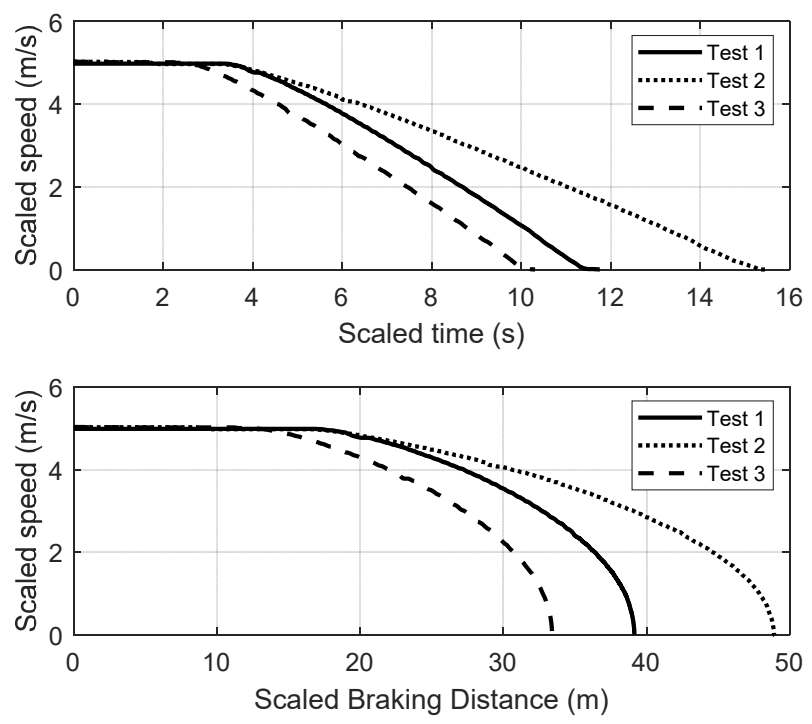

Figure 6: Scaled values of speed and time during the braking manoeuvres (up) and scaled braking curves in all three tests (down).

is approximately the same for tests 1 and 2, which are performed with a similar pressure reduction ramp, while a lower time is required in test 3 , which is carried out with the fastest ramp. When the brake cylinder pressure reaches the limit value set for the test, a linear trend of speed reduction in time can be noticed in all three tests, i.e., the wheelset is braked with an approximately constant deceleration.

Moreover, Fig. 6 highlights that the minimum barking distance is obtained in test 3, which is carried out with the same limit value as test 1, but with a faster ramp. At the same time, the comparison of tests 2 and 3, which are performed with the same ramp but different limit values of brake cylinder pressure, shows that the braking distance and time are higher for test 2 , which has a lower saturation value of brake cylinder pressure and is thus braked with reduced forces.

The preliminary tests shown in this section are not reproductions of a real braking manoeuvre for a railway freight vehicle. In fact, the influence of several parts of railway braking system was neglected in the experimental activity. Moreover, the pressure reduction in the main brake pipe is typically not linear in time, as registered in several experimental works. However, data obtained from this experimental campaign were in total agreement with the expectations, since higher ramps and higher values of maximum pressure in the brake cylinder led to reduced braking times and distances. Therefore, the preliminary experimental activity described in the paper can be considered as successful, since the new arrangement of the multi-axle roller-rig proved a suitable solution for both the reproduction of the braking manoeuvres of the railway vehicles and the acquisition of the signals usually required by braking system monitoring algorithms, which are acquired by the monitoring unit.

\section{CONCLUSIONS}

The paper describes an experimental activity aiming to demonstrate the feasibility of using an existing multi-axle roller-rig as a laboratory device for the development and testing of new 
railway braking plant monitoring systems. The roller-rig had been originally designed to investigate degraded adhesion conditions and adhesion recovery phenomena occurring during braking operations. Therefore, a few changes to the original arrangement were made in order to adapt the test bench to the new experimental activity. First, a pneumatic plant, reproducing the main features of the typical air brake system installed on freight trains, was specifically designed. Moreover, a new test bench control software was developed and implemented in LabVIEW. This software is provided with an algorithm able to reduce the roller speed according to the measured braking force, thus reproducing the vehicle inertia on the roller shaft. At the same time, the control software manages data acquisition and transmission to a monitoring unit, which acquires the same signals as required by typical concepts of railway braking plant monitoring systems.

Some preliminary tests, concerning the reproduction of simplified braking operations, were performed on the new arrangement of the bench and the results highlighted a good agreement with the expectations. Therefore, the goal of the activity can be considered as accomplished, since the new arrangement proved to be an effective solution to reproduce realistic railway braking operations by means of a laboratory device and to acquire with the monitoring unit the signals required by typical monitoring algorithms. In future work the current arrangement could thus be exploited to develop and test new air brake plant monitoring systems and algorithms.

The future developments of the experimental activity could aim to reduce the discrepancies between the current arrangement of the bench and the real freight vehicles air brake system. For instance, the current disc brakes could be replaced by a mechanical system with brake blocks and eventually a brake rig, while changes to the pneumatic system or to the control software could reproduce the influence of the relay valve. Finally, future tests could be performed using experimental data of pressures in the brake plant and vehicle speed, eventually investigating the behaviour of monitoring algorithms in simulated failure mode.

\section{REFERENCES}

[1] EN, 50343: Railway applications - Rolling stock - Rules for installation of cabling, 2014.

[2] De Pasquale, G., Somà, A. \& Zampieri, N., Design, simulation, and testing of energy harvesters with magnetic suspensions for the generation of electricity from freight train vibrations. Journal of Computational and Nonlinear Dynamics, 7(4), 2012.

[3] Bosso, N., Magelli, M. \& Zampieri, N., Application of low-power energy harvesting solutions in the railway field: a review. Vehicle System Dynamics, pp. 1-31, 2020.

[4] Bernal, E., Spiryagin, M. \& Cole, C., Onboard condition monitoring sensors, systems and techniques for freight railway vehicles: A review. IEEE Sensors Journal, 19(1), pp. 4-24, 2019.

[5] Li, C., Luo, S., Cole, C. \& Spiryagin, M., An overview: modern techniques for railway vehicle on-board health monitoring systems. Vehicle System Dynamics, 55(7), pp. 1045-1070, 2017.

[6] Bosso, N., Gugliotta, A. \& Zampieri, N., Wheel flat detection algorithm for onboard diagnostic. Measurement, 123, pp. 193-202, 2018.

[7] Somà, A., Aimar, M. \& Zanardelli, A., Experimental data measured with an on board unit for condition monitoring of freight wagons. Ingegneria Ferroviaria, 72(7-8), pp. 547-567, 2017.

[8] Zampieri, N., Bosso, N. \& Gugliotta, A., Innovative monitoring systems for onboard vehicle diagnostics. Civil-Comp Proceedings, 110, 2016. 
[9] Bosso, N., Gugliotta, A. \& Zampieri, N., Design and testing of an innovative monitoring system for railway vehicles. Proceedings of the Institution of Mechanical Engineers, Part F: Journal of Rail and Rapid Transit, 232(2), pp. 445-460, 2018.

[10] Bosso, N., Allen, P.D. \& Zampieri, N., Scale testing theory and approaches. Handbook of Railway Vehicle Dynamics, 2nd ed., CRC Press, pp. 825-867, 2019.

[11] Bosso, N., Gugliotta, A. \& Somà, A., Dynamic behavior of a railway wheelset on a roller rig versus tangent track. Shock and Vibration, 11(3-4), pp. 467-492, 2004.

[12] Bosso, N., Gugliotta, A. \& Zampieri, N., Study of the wheel-rail adhesion under degraded conditions using a multi-axles roller-rig. Dynamics of Vehicles on Roads and Tracks, vol. 2, CRC Press, pp. 803-808, 2017.

[13] Bosso, N., Gugliotta, A., Magelli, M., Oresta, I.F. \& Zampieri, N., Study of wheel-rail adhesion during braking maneuvers. Procedia Structural Integrity, 24, pp. 680-691, 2019.

[14] Bosso, N. \& Zampieri, N., Experimental and numerical simulation of wheel-rail adhesion and wear using a scaled roller rig and a real-time contact code. Shock and Vibration, 2014.

[15] Bosso, N., Magelli, M. \& Zampieri, N., Investigation of adhesion recovery phenomenon using a scaled roller-rig. Vehicle System Dynamics, pp. 1-18, 2019.

[16] Bosso, N., Gugliotta, A. \& Zampieri, N., Strategies to simulate wheel-rail adhesion in degraded conditions using a roller-rig. Vehicle System Dynamics, 53(5), pp. 619-634, 2015.

[17] Bosso, N., Gugliotta, A. \& Zampieri, N., A test rig for multi-wheelset adhesion experiments. Civil-Comp Proceedings, 110, 2016.

[18] Bosso, N., Gugliotta, A., Magelli, M. \& Zampieri, N., Experimental setup of an innovative multi-axle roller rig for the investigation of the adhesion recovery phenomenon. Experimental Techniques, 43(6), pp. 695-706, 2019..

[19] UIC 541-1, Brakes - Regulations concerning the design of brake components, 2003.

[20] UIC 540, Brakes - Air brakes for freight trains and passenger trains, 2016.

[21] Bosso, N., Gugliotta, A., Magelli, M. \& Zampieri, N., Monitoring of railway freight vehicles using onboard systems. Procedia Structural Integrity, 24, pp. 692-705, 2019.

[22] Aboubakr, A.K., Shabana, A.A. \& Bosso, N., Numerical study of the noninertial systems: Application to train coupler systems. Nonlinear Dynamics, 68(1-2), pp. 215233, 2012.

[23] Wu, Q. et al., International benchmarking of longitudinal train dynamics simulators: results. Vehicle System Dynamics, 56(3), pp. 343-365, 2018.

[24] Bosso, N. \& Zampieri, N., Long train simulation using a multibody code. Vehicle System Dynamics, 55(4), pp. 552-570, 2017. 